\title{
Mutational Screening of PARKIN Identified a 3' UTR Variant (rs62637702) Associated with Parkinson's Disease
}

\author{
Lorena de Mena • LLuís Samaranch • Eliecer Coto • \\ Lucía F. Cardo • René Ribacoba • Oswaldo Lorenzo- \\ Betancor • Pau Pastor • Li Wang • Jaione Irigoyen • \\ Ignacio F. Mata • Marta Díaz • Germán Moris • \\ Manuel Menéndez • Ana I. Corao • Elena Lorenzo • \\ Victoria Alvarez
}

Received: 27 August 2012 /Accepted: 13 December 2012 /Published online: 30 December 2012

(C) Springer Science+Business Media New York 2012

\begin{abstract}
P R K N$ mutations have been linked to Parkinson's disease (PD). Most of the mutational screenings have focused on the coding exons. The $3^{\prime}$ untranslated region (UTR) could also harbor functionally relevant nucleotide changes. We performed a mutational screening of PRKN in a cohort of early-onset PD patients $(n=235)$ from Spain. We found 16 mutations (five new): 16 patients (7\%) carried two mutations and only one mutation was found in 28 (12\%). Patients with two mutations had significantly lower mean
\end{abstract}

Electronic supplementary material The online version of this article (doi:10.1007/s12031-012-9942-y) contains supplementary material, which is available to authorized users.

L. de M. and LL. S. contributed equally to this work.

L. de Mena $\cdot$ E. Coto $\cdot$ L. F. Cardo $\cdot$ M. Díaz $\cdot$ A. I. Corao $\cdot$

V. Alvarez

Genética Molecular-Laboratorio de Medicina,

Hospital Universitario Central de Asturias, Oviedo, Spain

L. Samaranch $\cdot$ O. Lorenzo-Betancor $\cdot$ P. Pastor $\cdot$ J. Irigoyen $\cdot$

E. Lorenzo

Neurogenetics Laboratory, Division of Neurosciences,

Center for Applied Medical Research, (CIMA),

University of Navarra, Pamplona, Spain

R. Ribacoba $\cdot$ G. Moris $\cdot$ M. Menéndez

Neurología, Hospitales Universitario Central Asturias and Álvarez

Buylla-Mieres, Asturias, Spain

E. Coto

Department of Medicine, University of Oviedo,

Oviedo, Spain

O. Lorenzo-Betancor $\cdot$ P. Pastor $\cdot$ J. Irigoyen $\cdot$ E. Lorenzo Department of Neurology, Clínica Universidad de Navarra,

University of Navarra School of Medicine, Pamplona, Spain age (30 \pm 9 years) compared to patients with one $(40 \pm 7)$ or no mutation $(42 \pm 7)$. We found a total of 15 nucleotide variants (three new) in the $3^{\prime}$ UTR region. The frequency of carriers of the rare $r s 62637702 \mathrm{G}$ allele $(* 94 \mathrm{~A} / \mathrm{G})$ was significantly lower among the patients compared to healthy controls $(n=418)$ ( 0.03 vs. $0.004 ; p<0.001)$, suggesting a protective role for this allele. In order to investigate the basal effect of this variant, we performed luciferase assays. No different basal activity was observed between the two

L. Wang

Departments of Medicine and Oncological Sciences,

Huntsman Cancer Institute, University of Utah School of Medicine,

Salt Lake City, UT, USA

\section{F. Mata}

Geriatric Research, Education and Clinical Center,

Veterans Affairs Puget Sound Health Care System,

Seattle, WA, USA

\section{F. Mata}

Department of Neurology,

University of Washington School of Medicine,

Seattle, WA, USA

V. Alvarez ( $\square)$

Genética Molecular, Hospital Central Asturias-Maternidad,

33006, Oviedo, Spain

e-mail: victoria.alvarez@sespa.princast.es 
alleles. In conclusion, the rs62637702 polymorphism was associated with PD. This could be a surrogate marker for disease risk, in linkage disequilibrium with other nonidentified functional variant.

Keywords Parkinson's disease $\cdot$ Parkin $\cdot$ Mutation $\cdot$ DNA polymorphisms $\cdot$ Genetic risk $\cdot$ Genetics

\section{Introduction}

Several genes have been linked to autosomal dominant and recessive forms of Parkinson's disease (PD) (Vila and Przedborski 2004). PRKN (PARK2 locus, OMIM*602544) maps to chromosome region 6q35.3-q27.1 and encodes an E3 ubiquitin protein ligase. PRKN mutations have been found in $10-20 \%$ of the patients with early-onset PD (EOPD), mainly homozygous or compound heterozygous for PRKN mutations (Alvarez et al. 2001; Hedrich et al. 2002). However, most of the mutational screenings also reported a large amount of patients with only one identified mutated allele. Based on these findings, a dominant effect with variable penetrance has been proposed for some PRKN mutations (Sun et al. 2006; Lesage et al. 2008). These mutational studies analyzed the coding exons and a few intron flanking nucleotides, and the presence of a second non-identified PRKN mutation outside this regions could not be excluded. Nucleotide changes in the $3^{\prime}$ untranslated region ( $3^{\prime}$ UTR) of several genes have been linked to the risk of developing several diseases, including PD (Wang et al. 2008; Li et al. 2012; Papagregoriou et al. 2012). The pathogenic mechanism for these $3^{\prime}$ UTR variants would implicate the differential binding of micro RNAs (miRNAs) (Hebert and De Strooper 2007; Wang et al. 2008).

Our aim was to contribute in defining the mutational spectrum of $P R K N$. For this purpose, we analyzed the coding exons and the 3' UTR in a cohort of Spanish PD patients.

\section{Materials and Methods}

Patients and Controls

We searched for PRKN mutations in a total of 235 EOPD patients (mean age at diagnosis $41 \pm 8$ years; $65 \%$ male) (Supplementary Table 1). PD was diagnosed according to the UK Parkinson's Disease Society Brain Bank Clinical Criteria (Hughes et al. 1992). EOPD was considered as an onset of symptoms at age $\leq 50$ years (see www.ninds.nih. gov/disorders/parkinsons_disease). Patients who had at least one first-degree PD affected relative were classified as familial cases. The controls were 418 healthy individuals $(140 \leq 50$ years; $278>50$ years $)$ from the same regions (Asturias and Navarre, Northern Spain). The rs62637702 single nucleotide polymorphism (SNP) was also genotyped in a total of 306 late-onset (LOPD) patients (mean onset age $66 \pm 9$ years; $51 \%$ male).

All the patients/controls gave their informed consent to participate in the study, approved by the Ethical Committees of Hospital Central Asturias and Clínica Universidad de Navarra.

\section{PRKN Sequencing}

Genomic DNA was obtained from blood leukocytes. The 12 $P R K N$ exons were polymerase chain reaction (PCR)-amplified in the 235 EOPD patients with primers designated from the intronic sequences (Supplementary Table 2). PCR fragments were sequenced with BigDye chemistry in an ABI3130xl equipment (Applied Biosystems, Foster City, CA, USA). All the new (non-reported) putative mutations were screened in the 418 healthy controls through single strand conformation analysis (SSCA) of the corresponding PCR fragments (Supplementary Fig. 1). The putative functional effect of intronic nucleotide changes on pre-mRNA splicing was determined online (http://www.fruitfly.org/ seq_tools/splice.html).

\section{PRKN Exon Rearrangements}

Patients with only one sequencing mutation $(n=28)$ or no mutations $(n=191)$ were screened for PRKN exon rearrangements through multiple ligation-dependent probe amplification (MLPA) assay (P052 and P052B SALSA MLPA Parkinson 2 probemix kit, MRC-Holland, Amsterdam, The Netherlands).

\section{PRKN 3' UTR Analysis}

The 3' UTR of PRKN was amplified (ten overlapping PCR fragments) in all the patients and controls and the presence of DNA variants determined through SSCA (Supplementary Table 2 and Supplementary Fig. 1). Individuals with different SSCA patterns were sequenced to characterize the nucleotide variants. Two online tools (targetscan.org and microRNA.org) were used to determine whether SNP rs62637702 could affect a miRNA binding site.

\section{Luciferase Reporter Assay}

Two luciferase reporter plasmids containing the PRKN 3' UTR sequence and differing only by the rs62637702 SNP were constructed. The two reporter plasmids were constructed by inserting the PCR fragments from two patients with primers GGAGCACTAGTATACCCAGTGTC TACCTTCATTTT (forward) and CATAAGCTTGT GAGACTCATGCCCTCAGTT (reverse) into the SpeI/ HindIII sites in the $3^{\prime}$ of the luciferase gene of the pmiR- 
Table $1 P R K N$ mutations found in the coding exons and intronic flanking nucleotides

\begin{tabular}{llll}
\hline Mutation & Exon/intron & Variant ID & Type \\
\hline p.Q34fs & Exon 2 & new & Frameshift \\
c.225delA & Exon 2 & known & Frameshift \\
Exon rearrangement & Exon 2 & known & Deletion \\
Exon rearrangement & Exon 3 & known & Deletion \\
Exon rearrangement & Exon4 & known & Deletion \\
Exon rearrangement & Exon2-3 & known & Deletion \\
Exon rearrangement & Exon 3-5 & known & Duplication \\
c.461delG & Exon 4 & New & Frameshift \\
M192L & Exon 5 & known & Missense \\
P202L & Exon 5 & New & Missense \\
C212Y & Exon 6 & Rs137853058 & Missense \\
T240M & Exon 6 & Rs137853054 & Missense \\
R275W & Exon 7 & Rs34424986 & Missense \\
c.1175-1176 delGA & Exon 11 & known & Frameshift \\
c.1203-1204 delTC & Exon 11 & New & Frameshift \\
R402C & Exon 11 & Rs55830907 & Missense \\
Ivs11 -3bpC/G & Intron 11 & known & Splicing \\
G430S & Exon 12 & Rs191486604 & Missense \\
P437L & Exon 12 & Rs149953814 & Missense \\
W445.Stop & Exon 12 & known & Frameshift \\
V456I & Exon12 & New & Missense \\
\hline
\end{tabular}

Report vector (Ambion, Austin, TX). Twenty-four hours before transfection $6 \times 10^{4}$ HEK 293 cells per well were plated in a 24 well plate. Each well was transfected with $150 \mathrm{ng}$ of $P R K N-3^{\prime}$ UTR-luciferase construct and $50 \mathrm{ng}$ of Renilla control vector. After $48 \mathrm{~h}$, the assays were performed using the DualLuciferase $^{\circledR}$ reporter assay system (Promega Corp., Madison, WI). Basal luciferase activities were normalized to Renilla activities for each well transfected. The experiment was performed in triplicate, and each well was assayed in triplicate.

\section{Statistical Analysis}

Differences between 3' UTR allele and genotype frequencies were compared through Chi-squared or Fisher's exact tests. These were also used to determine whether the observed genotype frequencies differed from those expected under the Hardy-Weinberg equilibrium. The Student's $t$ and ANOVA were used to compare the mean age between the groups. Basal luciferase activities were expressed as means $\pm \mathrm{SD}$ and differences, compared through ANOVA. All the statistical analysis were performed with the R-project free software (http://www.R-project.org), and a $p$ value $<0.05$ was considered as statistically significant.

The linkage disequilibrium for 3' UTR SNPs and difference between haplotype frequencies were determined as reported (Barrett et al. 2005; Gabriel et al. 2002).

\section{Results \\ PRKN Mutations}

The sequencing of the PRKN coding exons + intronic flanking nucleotides identified a total of 16 mutations, five of them new (Table 1). All the new mutations gave typical SSCA patterns that were absent in the controls (Supplementary Fig. 1). Allele and genotype frequencies did not differ between patients and controls for the two common missense polymorphisms S167N and D394N.

We found two mutations in 15 patients $(6.4 \%)$ and only one in $12(5.5 \%)$ patients (Supplementary Table 3). PD subjects with only one mutation or without an identified PRKN mutation were screened for large gene rearrangements. Duplications of exons 2, 3, 2-3, or 2-4, and deletions of exons 4 or 3-4 were found in 17 patients (Supplementary Fig. 2). Only one was compound heterozygous for an exon 4 deletion and a single nucleotide mutation $(\mathrm{T} 240>\mathrm{M})$. Thus, we identified two $P R K N$ mutations in $16(7 \%)$ and only one in $28(12 \%)$ patients. Patients with two PRKN mutations had a significantly lower mean onset age $(30.25 \pm 8.8$ years $)$ compared to patients with only one $(39.75 \pm 7.2$ years; $p<$ $0.001)$ and without mutations $(42.02 \pm 6.9$ years; $p<0.001)$. Two mutations were also significantly more frequent among familial vs. sporadic patients $(p=0.045)$ (Table 2$)$.

\section{PRKN 3' UTR Analysis}

We identified 15 nucleotide variants in the PRKN 3' UTR, 12 of them reported SNPs (Supplementary Table 4). All

Table 2 Mean age at diagnosis and known family history in patients according to the PRKN mutation status

\begin{tabular}{lcccc}
\hline & Sex $(\%$ male $)$ & Family history $N(\%)$ & $p$ value $^{\mathrm{a}}$ & Mean age \\
\hline Non-mutated $(N=191)$ & $122(63.9)$ & $59(30.9)$ & & $42.02 \pm 6.9$ \\
Homozygous $(N=16)$ & $13(81.3)$ & $9(56.3)$ & 0.045 & $30.25 \pm 8.8$ \\
Heterozygous $(N=28)$ & $17(60.7)$ & $12(42.9)$ & 0.210 & $39.75 \pm 7.7$ \\
\hline
\end{tabular}

${ }^{\text {a }}$ Non- $P R K N$ mutation carriers vs. mutation carriers

${ }^{\mathrm{b}}$ Mean age non-mutation carriers vs. mutation carriers 
Table 3 Rs62637702 genotype and allele frequencies in patients and healthy controls

\begin{tabular}{|c|c|c|c|c|c|c|}
\hline & $\begin{array}{l}\text { EOPD } \\
(n=235)\end{array}$ & $\begin{array}{l}\text { Controls } \\
\leq 50 \text { years } \\
N=140\end{array}$ & $\begin{array}{l}\text { LOPD } \\
(n=306)\end{array}$ & $\begin{array}{l}\text { Controls } \\
>50 \text { years } \\
N=278\end{array}$ & $\begin{array}{l}\text { Total patients* } \\
N=541\end{array}$ & $\begin{array}{l}\text { Total controls* } \\
N=418\end{array}$ \\
\hline AA & $233(99 \%)$ & $135(96 \%)$ & $303(99 \%)$ & $261(94$ \%) & $536(99 \%)$ & 396 (95 \%) \\
\hline $\mathrm{AG}$ & $2(1 \%)$ & $5(4 \%)$ & $3(1 \%)$ & $17(6 \%)$ & $5(1 \%)$ & $22(5 \%)$ \\
\hline GG & 0 & 0 & 0 & 0 & 0 & 0 \\
\hline A & $468(99 \%)$ & $275(98 \%)$ & $609(99 \%)$ & $539(97$ \%) & $1,072(99 \%)$ & $814(97$ \%) \\
\hline $\mathrm{G}$ & $2(<1 \%)$ & $5(2 \%)$ & $3(<1 \%)$ & $17(3 \%)$ & $5(<1 \%)$ & $22(3 \%)$ \\
\hline
\end{tabular}

${ }^{*} p<0.001$ (G carriers, total PD vs. total controls)

these variants gave a characteristic SSCA pattern and were thus SSCA-genotyped in the healthy controls. Two of the $3^{\prime}$ UTR variants (*93-94 Ins AC and *180-238 duplication) were found in two different patients (negative for PRKN mutations) and none of the controls. Only the rs62637702 $(* 94 \mathrm{~A} / \mathrm{G})$ allele and genotype frequencies were significantly different between the two groups. Compared to EOPD patients, carriers of the rare $\mathrm{G}$ allele were more frequent in age-matched controls ( $\leq 50$ years) and total controls, suggesting a protective effect against EOPD in our population (Table 3). To determine whether this SNP was also associated with the risk of developing LOPD, we genotyped 306 patients $>50$ years (onset age) and found that G-carriers were also more frequent in healthy controls than in LOPD (22/418, $5.3 \%$ vs. 3/306, $1 \% ; p=0.001)$. No significant difference was found between the younger ( $\leq 50$ years) and older ( $>50$ years) controls. None of the G-carrier patients had PRKN mutations.

We determined the linkage disequilibrium between rs62637702 and the other 3' UTR SNPs in our population
(Supplementary Fig. 3). No significant difference was found for the haplotype frequencies, patients vs. controls (data not shown).

We used two public online tools (targetscan.org and microRNA.org) to determine whether this SNP could affect a miRNA binding site, and none of them predicted that any putative miRNA site could be affected by this SNP. To determine whether rs62637702 could affect basal gene expression, we cloned PRKN 3' UTR fragments containing either $\mathrm{A}$ or $\mathrm{G}$ in luciferase reporter plasmids and transfected and measured the luciferase activity in HEK-293 cells. No significant differences were found between the two constructs (Fig. 1).

\section{Discussion}

We reported the mutational spectrum of $P R K N$ in a cohort of Spanish EOPD patients. In addition to sequencing the whole coding exons and immediately flanking intronic nucleotides,
Fig. 1 Relative luciferase expression of the two $3^{\prime}$ UTR constructs for rs 62637702 alleles. a Scheme of the PARK2 3' UTR-luciferase constructs containing the two rs62637702 alleles. b HEK 293 cells where transfected with the two constructs and harvested $48 \mathrm{~h}$ after transfection. Luciferase activity was normalized with Renilla activity. $p=0.4$
(A)

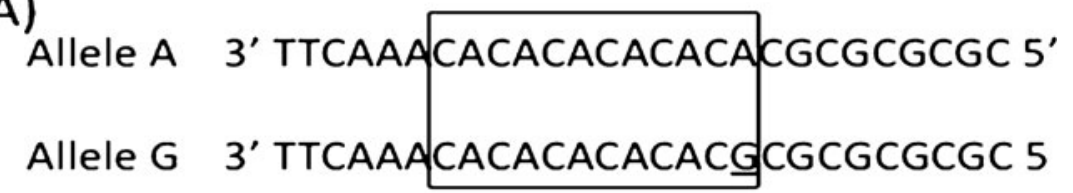

(B)

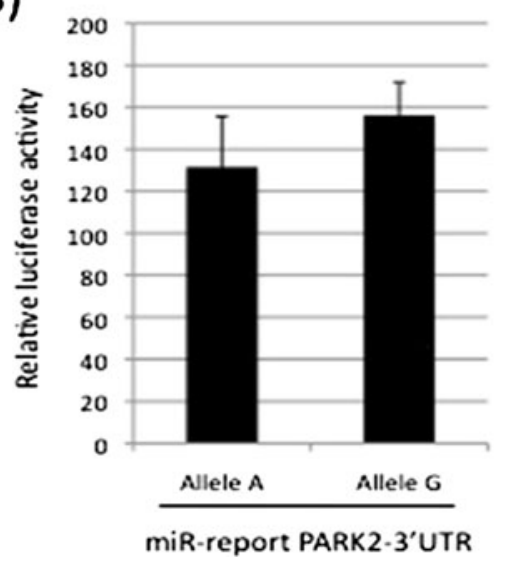


we also performed large gene duplication/deletion scanning. Our study was, thus, more complete than others that did not determined the presence of gene rearrangements. The frequency of patients with two (7\%) and one (12\%) mutation was similar to that reported by others (Hedrich et al. 2002; Oliveira et al. 2003; Periquet et al. 2003; Sironi et al. 2008; Koziorowski et al. 2009). In agreement with other studies, we also found a significantly lower mean onset age among patients with two mutations (Sironi et al. 2008).

To our knowledge, this is the first report describing the PRKN 3' UTR variation in a cohort of EOPD patients. Because they did not encode for amino acids or affect premRNA splicing, the $3^{\prime}$ UTRs have been rarely included in mutational screenings. This scenario has changed after the discovery of its role in translational regulation by microRNA (miRNA) binding. Through this mechanism, miRNAs regulate many cellular processes, and the deregulation of miRNA pathways could result in neurodegeneration and PD (Kosik 2006; Hebert and De Strooper 2007; Wang et al. 2008; de Mena et al. 2010). Some miRNAs have been implicated in the post-transcriptional regulation of PDassociated genes such as SNCA, and common 3' UTR variants in other genes could regulate the risk for PD (Junn et al. 2009; Doxakis 2010).

Only two rare 3' UTR changes (*93-94 Ins AC and *180238 duplication) were found in two patients and absent among the controls. The main novelty in our study was a significant association between a rare SNP $(* 94 \mathrm{~A} / \mathrm{G}$; rs62637702) and PD. G-carriers were more frequent among healthy controls, suggesting that this allele could have a protective effect against PD in our population. The protective effect was also supported by the higher frequency of the G-allele carriers among elderly compared to the younger controls ( 6 vs. $4 \%$ ), although the difference was non-significant. Sironi et al. found the rare $\mathrm{G}$ variant in three out of $146 \mathrm{EOPD}(2 \%)$ and two out of 50 controls (4\%) (Sironi et al 2008). Patients and controls in this study had a younger mean age compared to ours, and only 50 controls were genotyped by these authors. This could explain the differences in $* 94 \mathrm{G}$ frequencies between the two studies. Bardien and colleagues also found this variant in EOPD, but the frequency in controls was not determined (Bardien et al. 2009).

The *94 A/G SNP could be directly linked to PD risk through a functional effect on parkin expression mediated by changes in a miRNA binding site. However, none of the two alleles was predicted to affect the binding of miRNAs. In addition, we did not find significant differences in luciferase basal activities between plasmid constructs differing by rs62637702. The lack of a direct effect for this SNP suggested that other gene variants in linkage disequilibrium with rs62637702 could explain the observed association with PD risk. Because no missense polymorphism linked to EOPD was identified, we can conclude that the observed effect of this SNP was due to linkage disequilibrium with a functional variant in the coding region. However, we cannot exclude that rs62637702 was a surrogate marker for a functional variant in the promoter or internal intronic nucleotides.

In conclusion, we reported the mutational spectrum of PRKN/PARK2 gene in a cohort of Spanish EOPD patients. In addition, our screening of the $3^{\prime}$ UTR identified a significant association of SNP rs62637702 with the risk of developing PD. Further studies should be necessary to confirm this association in other populations and to clarify the functional effect of this and other linked PRKN variants.

Acknowledgments The authors thank the Fundacion Parkinson Asturias and Obra Social Cajastur for their support. This work was supported by grants from the Spanish Fondo de Investigaciones Sanitarias-Fondos FEDER European Union (FIS-05/008, 08/0915, and 11/0093). LFC and LDM are predoctoral fellowships of FICYT-Principado de Asturias. LS held a Torres Quevedo fellowship from the Spanish Ministry of Science and Technology, co-financed by the European Social Fund. This study was supported by a grant from the Spanish Ministry of Education and Science (SAF2006-10126: 2006-2009), by the project 061131 from the Fundació La Marató de TV3 and by the UTE project FIMA, Spain to PP. We thank all participating subjects and their relatives for their contribution to the study. We thank Drs. Maria A. Pastor, Jose Obeso, Maria R Luquin, Maria C Rodríguez-Oroz, and Mario Riverol for recruiting some subjects for the study.

Conflict of Interests All the authors declare no conflicts of interest related with this work.

\section{References}

Alvarez V, Guisasola LM, Moreira VG, Lahoz CH, Coto E (2001) Early-onset Parkinson's disease associated with a new parkin mutation in a Spanish family. Neurosci Lett 313(1-2):108-110

Bardien S, Keyser R, Yako Y, Lombard D, Carr J (2009) Molecular analysis of the parkin gene in South African patients diagnosed with Parkinson's disease. Parkinsonism Relat Disord 15(2):116 121

Barrett JC, Fry B, Maller J, Daly MJ (2005) Haploview: analysis and visualization of $\mathrm{LD}$ and haplotype maps. Bioinformatics 21 (2):263-265

de Mena L, Coto E, Cardo LF et al (2010) Analysis of the Micro-RNA133 and PITX3 genes in Parkinson's disease. Am J Med Genet B Neuropsychiatr Genet

Doxakis E (2010) Post-transcriptional regulation of alpha-synuclein expression by mir-7 and mir-153. J Biol Chem 285(17):1272612734

Gabriel SB, Schaffner SF, Nguyen H et al (2002) The structure of haplotype blocks in the human genome. Science 296(5576):2225-2229

Hebert SS, De Strooper B (2007) Molecular biology. miRNAs in neurodegeneration. Science 317(5842):1179-1180

Hedrich K, Marder K, Harris J et al (2002) Evaluation of 50 probands with early-onset Parkinson's disease for Parkin mutations. Neurology 58(8):1239-1246

Hughes AJ, Daniel SE, Kilford L, Lees AJ (1992) Accuracy of clinical diagnosis of idiopathic Parkinson's disease: a clinico-pathological study of 100 cases. J Neurol Neurosurg Psychiatry 55(3):181-184

Junn E, Lee KW, Jeong BS, Chan TW, Im JY, Mouradian MM (2009) Repression of alpha-synuclein expression and toxicity 
by microRNA-7. Proc Natl Acad Sci U S A 106(31):1305213057

Kosik KS (2006) The neuronal microRNA system. Nat Rev Neurosci 7 (12):911-920

Koziorowski D, Hoffman-Zacharska D, Slawek J et al (2009) Low frequency of the PARK2 gene mutations in Polish patients with the early-onset form of Parkinson disease. Parkinsonism Relat Disord 16(2):136-138

Lesage S, Lohmann E, Tison F, Durif F, Dürr A, Brice A (2008) Rare heterozygous parkin variants in French early-onset Parkinson disease patients and controls. J Med Genet 45:43-46

Li H, Ren C, Fan Z et al (2012) A genetic variant in 3'-untranslated region of cyclooxygenases- 2 gene is associated with risk of gastric cancer in a Chinese population. DNA Cell Biol 31(7):1252-1257

Oliveira SA, Scott WK, Martin ER et al (2003) Parkin mutations and susceptibility alleles in late-onset Parkinson's disease. Ann Neurol 53(5):624-629
Papagregoriou G, Erguler K, Dweep H et al (2012) A miR-1207-5p binding site polymorphism abolishes regulation of HBEGF and is associated with disease severity in CFHR5 nephropathy. PLoS One 7(2):e31021

Periquet M, Latouche M, Lohmann E et al (2003) Parkin mutations are frequent in patients with isolated early-onset parkinsonism. Brain 126(Pt 6):1271-1278

Sironi F, Primignani P, Zini M et al (2008) Parkin analysis in early onset Parkinson's disease. Parkinsonism Relat Disord 14(4):326-333

Sun M, Latourelle JC, Wooten GF et al (2006) Influence of heterozygosity for parkin mutation on onset age in familial Parkinson disease: the GenePD study. Arch Neurol 63(6):826-832

Vila M, Przedborski S (2004) Genetic clues to the pathogenesis of Parkinson's disease. Nat Med 10(Suppl):S58-S62

Wang G, van der Walt JM, Mayhew G et al (2008) Variation in the miRNA-433 binding site of FGF20 confers risk for Parkinson disease by overexpression of alpha-synuclein. Am J Hum Genet 82(2):283-289 